\title{
Complete response of a highly advanced gastric carcinoma to preoperative chemoradiotherapy with $S-1$ and low-dose cisplatin
}

\author{
Nobunari Yoshimizu ${ }^{1}$, Yoshiro Saikawa ${ }^{1}$, Tetsuro Kubota ${ }^{1}$, Yasutada Akiba ${ }^{2}$, Masashi Yoshida ${ }^{1}$, \\ Yoshinide Otani $^{1}$, Koichiro Kumai ${ }^{1}$, Toshinumi Hibi $^{2}$, and Masaki Kitajima ${ }^{1}$ \\ ${ }^{1}$ Department of Surgery, School of Medicine, Keio University, 35 Shinanomachi, Shinjuku-ku, Tokyo 160-8582, Japan \\ ${ }^{2}$ Department of Internal Medicine, School of Medicine, Keio University, Tokyo, Japan
}

\begin{abstract}
S-1 has been developed as a new oral anticancer drug, based on the biological modulation of 5-fluorouracil. We report a patient with highly advanced gastric carcinoma who was treated successfully with a new combination chemoradiotherapy using S-1 and cisplatin (CDDP). The patient was a 37-year-old man who was diagnosed with advanced gastric carcinoma (T4N3M0) that had invaded the diaphragm and the paraaortic tissues. Remarkable tumor reduction was observed in the primary tumor and metastatic lymph nodes around the stomach after three cycles of the therapy. Radiological examination before surgery determined that a partial response (PR) had been achieved by the initial therapy. Adverse effects included only a gastrointestinal disorder that was limited to grade 2 when low-dose CDDP was utilized in the regimen, while an initial high dose of CDDP resulted in grade 3 toxicity, due to myelosuppression. The patient underwent curative surgery, including total gastrectomy, D2 lymph node dissection, and splenectomy, after completion of the radiochemotherapy regimen. No surgical complication was observed. No tumor cells were detected by pathological evaluation of the resected stomach and all the regional lymph nodes, confirming a pathological complete response (CR; grade 3 ). This regimen is a potent treatment for advanced gastric carcinoma, especially when used as preoperative chemotherapy to control cancer cells.
\end{abstract}

Key words Complete response · Gastric cancer · S-1 · Cisplatin $\cdot$ Radiation

\section{Introduction}

Improvements in diagnostic and cancer screening techniques in developed countries over the past decade have led to a dramatic increase in the detection of early

Offprint requests to: Y. Saikawa

Received: January 6, 2003 / Accepted: April 8, 2003 gastric cancer, compared with advanced gastric cancer. However, cases of highly advanced gastric carcinoma (incurable, unresectable, or inoperable) are still encountered in clinics. In general, curative surgery is believed to be the most desirable treatment for gastric carcinomas at present. Evaluations of clinical outcomes have indicated a poor prognosis for advanced gastric carcinoma patients who underwent macroscopically curative resection [1-3], with fewer than $50 \%$ of patients surviving for 5 years. This suggests that surgical treatment alone cannot control the growth of microscopic cancer cells, resulting in metastasis or dissemination in vivo through the surgical procedure. On the other hand, beneficial outcomes for gastric carcinoma patients were achieved through the use of adjuvant chemoradiotherapy along with surgical treatment. This was proven by randomized controlled trials carried out by Macdonald et al. [4], and several reports have also suggested the usefulness of chemotherapy against advanced gastric carcinoma [5-8].

There is a newly developed chemotherapy with the agent S-1, consisting of tegafur; gimeracil (5-chloro-2, 4dihydropyrimidine - as a dihydropyrimidine dehydrogenase inhibitor), which inhibits 5-fluorouracil (5FU) degradation; and oteracil (monopotassium 1, 2, 3, 4tetrahydro-2, 4-dioxo-1, 3, 5-triazine-6 carboxylateto reduce the gastrointestinal toxicity caused by the phosphorylation of 5FU) [9-11]. Several clinical trials involving the administration of S-1 alone for the treatment of measurable lesions of gastric carcinoma showed high response rates, of up to approximately $50 \%$ [12, 13], suggesting the possibility of novel effective regimens that would combine this therapy with other agents against gastric carcinoma. Because cisplatin (CDDP) has been clinically utilized with $5 \mathrm{FU}$ against gastric carcinoma worldwide, enhancing tumor toxicity [1416], CDDP was chosen as an agent for combined chemotherapy with S-1 against advanced gastric carcinoma. 
We report here a patient with highly advanced gastric carcinoma whose histological diagnosis confirmed his classification as showing a complete response (CR; cancer-free) after he received preoperative chemoradiotherapy that included S-1 and CDDP.

\section{Case report}

\section{Staging and classification}

The definitions of the Japanese Research Society for Gastric Cancer [17] were utilized in staging, radiological evaluation of the effects, and evaluation of histological efficacy. Combination chemotherapy with S-1 and lowdose CDDP (S-1/CDDP) was evaluated. The toxicity of S-1/CDDP was also classified using the Cancer Therapy
Evaluation Program, Common Toxicity Criterion, version 2.0 [18].

\section{Pretreatment diagnosis}

A 37-year-old man was admitted with the chief complaint of dyspnea with severe anemia. Physical examination did not indicate a specific problem. The serum hemoglobin level was $7.1 \mathrm{~g} / \mathrm{dl}$. The serum carcinoembryonic antigen (CEA) and carbohydrate antigen (CA)19-9 levels were $100.1 \mathrm{U} / \mathrm{ml}$ and $4121 \mathrm{U} / \mathrm{ml}$, respectively. Endoscopy of the upper gastrointestinal tract (gastrointestinal fiberscopy; GIF) demonstrated type 3 advanced gastric carcinoma in the cardia and a metastatic lesion in the esophagus (Fig. 1A,B). A biopsy specimen indicated that this was a poorly differentiated tubular adenocarcinoma. An abdominal computed to-
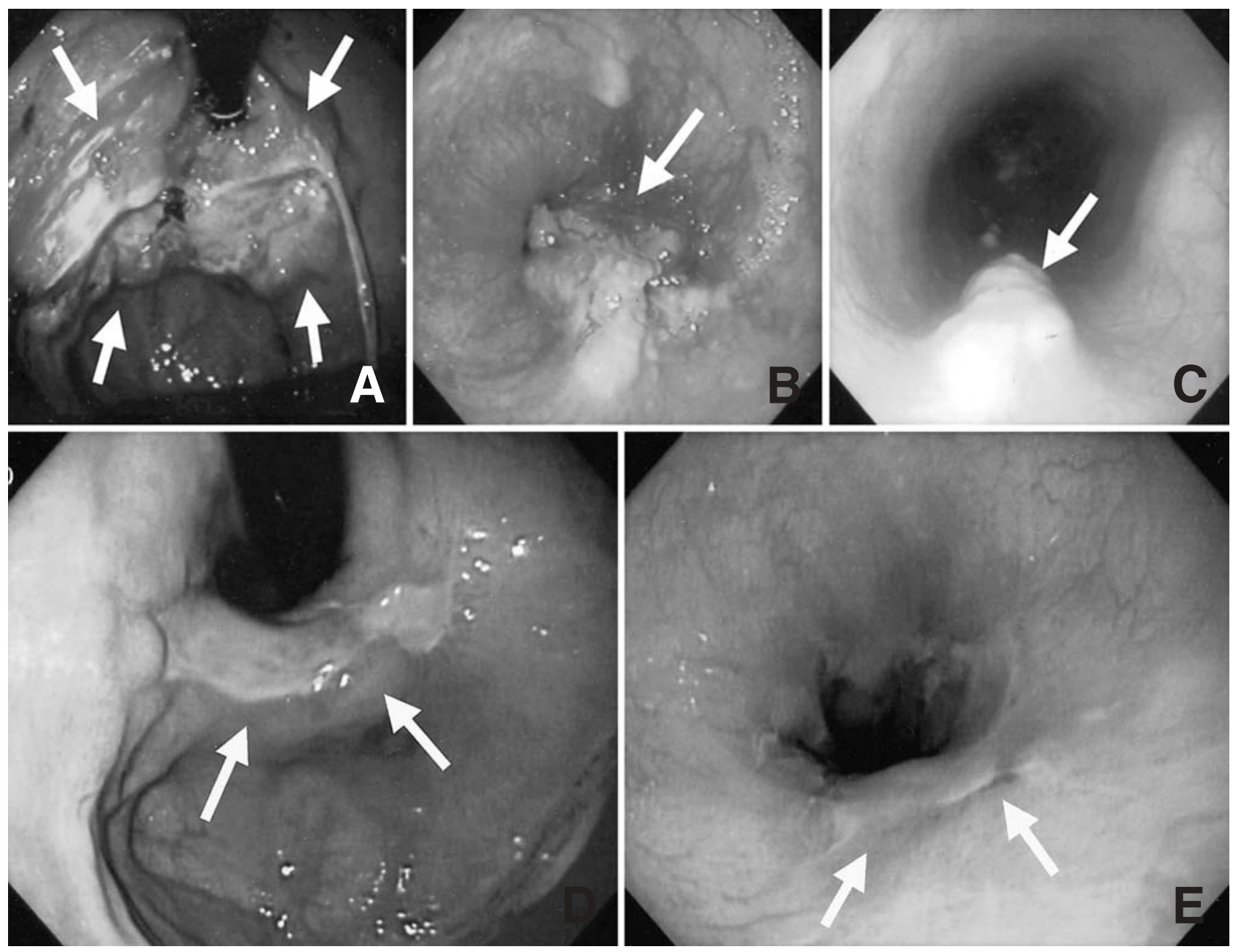

Fig. 1. A Gastrointestinal fiberoscopy (GIF) before chemotherapy showed an invasive tumor with ulceration causing stenosis (arrows). A diagnosis of type 3 advanced gastric carcinoma was made. B,C A metastatic tumor in the esophagus (arrow) was confirmed by pathological analysis to be a poorly differentiated adenocarcinoma, similar to the pri-

mary lesion. D GIF after chemotherapy demonstrated a decreased tumor volume and amelioration of the stenotic change (arrow). E GIF after chemotherapy showed disappearance of the esophageal tumor (arrows); no cancer cells were observed by endoscopic biopsy 

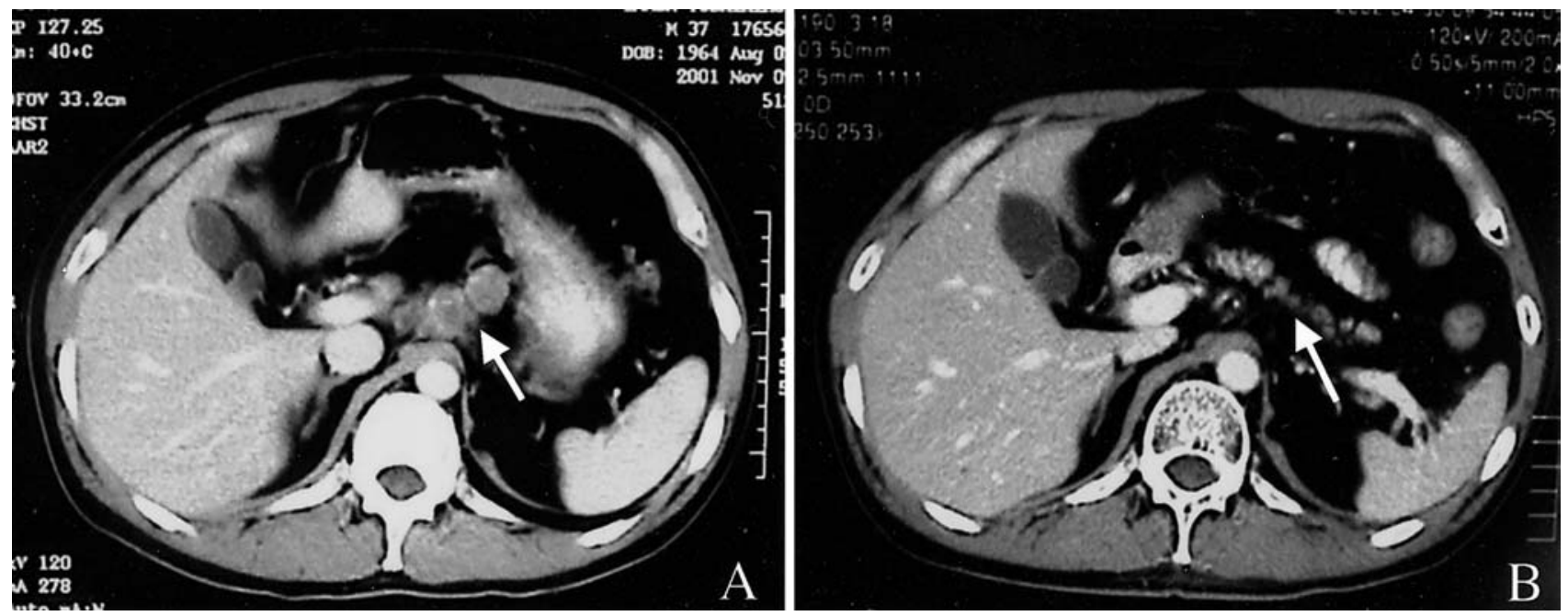

Fig. 2. A Abdominal computed tomography (CT) showed invasion of the cancer to the diaphragm and paraaortic tissues. Lymph nodes' metastasis was suspected in paraaortic lesion (arrow). This case was classified as stage IV (T4N3M0H0P0). B After three courses of the S1/cisplatin regimen, abdominal CT demonstrated a remarkable reduction of the lymph nodes (arrow)

mography (CT) scan showed that the gastric wall was thickened and the gastric tumor and giant lymph nodes around the stomach had invaded the diaphragm and the paraaortic tissues (Fig. 2A). The patient was diagnosed with stage IV advanced gastric carcinoma according to the Japanese classification of gastric carcinoma (cT4, $\mathrm{cN} 3, \mathrm{cH} 0, \mathrm{cP} 0$; c stage IV). The patient decided to undergo preoperative chemotherapy, carried out in an attempt to downstage the disease. This was done with the patient's informed consent in accordance with the advanced gastric carcinoma treatment protocols.

\section{Combination chemotherapy (S-1/CDDP) with radiation}

S-1 (Taiho Pharmaceutical, Tokyo, Japan) was prescribed as an orally administered drug, and CDDP was purchased from Nihon Kayaku (Tokyo, Japan).

S-1 was administered orally every day, at a dose of $80 \mathrm{mg} / \mathrm{m}^{2}$ per day, for 2 weeks, and simultaneously, $20 \mathrm{mg} / \mathrm{m}^{2}$ per day of CDDP was infused on days $1-5$, followed by a 2-day interval without CDDP for 2 weeks. Because the patient developed grade 3 leukocytopenia and grade 3 thrombocytopenia 2 weeks after the initiation of the chemotherapy (total dose: S-1, $1680 \mathrm{mg}$; CDDP, $320 \mathrm{mg}$, at that time), there was a 5-week interval without any drugs until the patient recovered from the toxicities. CDDP, at a decreased dose of $4 \mathrm{mg} / \mathrm{m}^{2}$ per day, was started again, with a decreased dose of S-1 $\left(60 \mathrm{mg} / \mathrm{m}^{2}\right.$ per day), with a 2-week drug-free interval after 3 weeks of drug administration ( 7 days of S-1 and 5 days of CDDP a week). The total doses of S-1 and
CDDP administered before surgery were $6120 \mathrm{mg}$ and $565 \mathrm{mg}$, respectively.

Radiation therapy ( $2 \mathrm{~Gy} /$ time per day) was added for the same period that CDDP was administered, with the same interval of drugs. The total dose of radiation was $60 \mathrm{~Gy}$ (given in 30 occasions). The irradiated area included the cardia of the stomach, and the esophagus in the thorax and abdomen.

\section{Radiological evaluation and toxicities}

The patient's response was evaluated after the chemoradiotherapy. GIF showed remarkable tumor regression and scar formation at the ulcerative lesion in the cardia, as well as the disappearance of the metastatic lesion in the esophagus, where no cancer cells were detected by endoscopic biopsy (Fig. 1D,E). Abdominal CT showed amelioration of the cancer invasion into the diaphragm. The lesions in the gastric regional and paraaortic lymph nodes had also disappeared. Radiological examination showed a partial response (PR) in the stomach and a complete response (CR) in lymph nodes (Fig. 2B) according to World Health Organization (WHO) criteria [19]. The serum CEA and CA19-9 levels were normalized after the therapy, to 4.5 and $20 \mathrm{U} / \mathrm{ml}$, compared with the pretherapy levels of 100.1 U/ml and $4121 \mathrm{U} / \mathrm{ml}$, respectively (Fig. 3). As for adverse effects, only gastrointestinal disorder was observed, and this was limited to grade 2 , when low-dose CDDP was utilized in the regimen, while an initial high dose of CDDP resulted in grade 3 toxicity due to myelosuppression. 


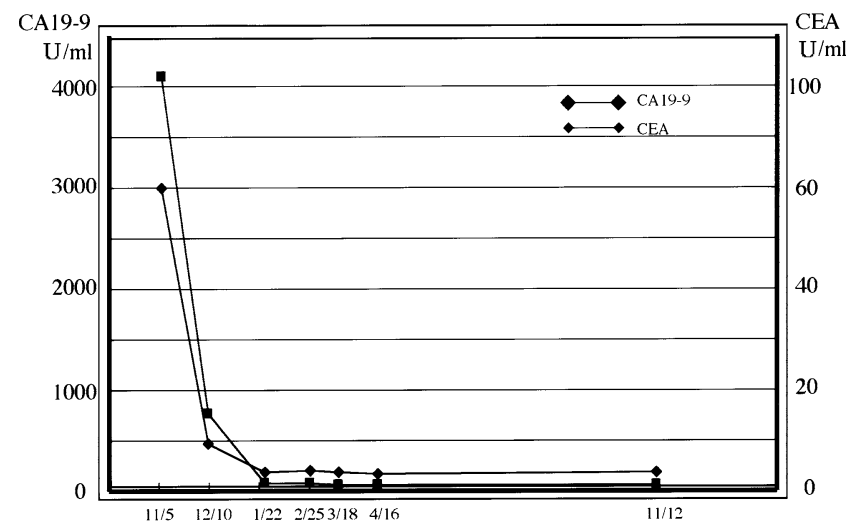

Fig. 3. Time course of serum carcinoembryonic antigen $(C E A)$ and carbohydrate antigen $(C A)$ 19-9 values. The high tumor marker values before therapy decreased remarkably after the chemoradiotherapy, and has been maintained within the normal range since the surgery was performed

\section{Intraoperative findings}

Laparotomy was used to detect a gastric tumor on the lesser curvature of the esophago-cardiac junction (ECJ). Lymph nodes around the lesser curvature of the stomach were swollen, while the paraaortic lymph nodes were not palpable. Cytology of the peritoneal washes was negative. Total gastrectomy, complete D2 lymph node dissection, and splenectomy were performed.

\section{Postoperative histological diagnosis}

Tumor pathological classifications and the grading of histological responses to the preoperative chemotherapy were based upon the General rules for the gastric cancer study in surgery and pathology in Japan [17]. Macroscopic ulcer scars were observed in the cardia (Fig. 4A), and no tumor cells were observed microscopically in any lesion of the primary tumor, in which the wall structure was completely replaced by scar tissues. The patient was thus diagnosed as showing a CR (Fig. 4B). In the regional lymph nodes, some of which were previously diagnosed with metastatic lesions, areas with strong fibrosis, lymphocytic infiltrations, and foreign body granuloma were observed, and this was also consistent with a diagnosis of CR (Fig. 4C).

\section{Postoperative course}

No postsurgical complications were observed, and the patient is alive without recurrence, 12 months after surgery and 17 months following the initial chemotherapy.

\section{Discussion}

Advanced gastric carcinomas that were treated with macroscopic curative surgery showed a 5-year survival rate of less than $50 \%$ in Western countries as well as in Japan [1-3], suggesting that surgical outcome alone cannot predict or prevent future recurrence or metastasis. We should therefore appreciate the difficulties of controlling "micro-cancer", which is forming or will be forming metastases, resulting in the dissemination of carcinoma cells. The development of additional strategies to be used in conjunction with surgery is urgently needed to overcome "micro-cancer" that is not eliminated by surgery.

The novel anticancer agent S-1, when given alone, showed a high response rate, of approximately $40 \%-$ $50 \%$, and low toxicity in several clinical studies of gastric cancer $[12,13]$. The clinical effects of 5FU are known to be enhanced by combination with CDDP in several kinds of carcinoma [15]. Previously, in a pilot study, we attempted to evaluate a new preoperative combination chemotherapy against highly advanced gastric carcinomas using S-1 and low-dose CDDP, and this resulted in a good response preoperatively in patients with stage IV gastric cancer [20]. In the study, six of ten patients who underwent surgical resection following the chemotherapy showed a pathological response above grade $1 \mathrm{~b}$ in either the stomach or lymph nodes. Further studies, including randomized controlled studies, will be necessary to evaluate the efficacy of the preoperative chemotherapy.

In the present patient, a comparatively high dose of CDDP with S-1 was administered, with the addition of radiation, which was administered because of the existence of a metastatic tumor in the patient's esophagus. This combined treatment resulted in complete responses in the primary lesion, the lymph nodes, and the metastatic esophageal tumor. Side effects of the therapy included the development of grade 3 myelosuppression, while low-dose CDDP $\left(4 \mathrm{mg} / \mathrm{m}^{2}\right.$ per day) did not result in such a severe adverse reaction in the patient, suggesting that additional improvements are needed in order to reduce the toxicity while maintaining the potent anticancer effect of the regimen that includes radiation. In our patient, the dose of CDDP was changed because of the severe toxicity caused by $20 \mathrm{mg} / \mathrm{m}^{2}$ per day of CDDP. While our previous study demonstrated that the dose of $6 \mathrm{mg} / \mathrm{m}^{2}$ per day of CDDP showed grade 2 toxicity of bone marrow suppression in almost all patients who received it in combination with S-1 [20], the recommended dose (RD) of CDDP is still under investigation for regimens with radiation, and a phase I/ II study for determining the RD of CDDP in such a regimen is necessary.

In recent years, patients with advanced gastric carcinomas with a CR, determined by radiological examina- 


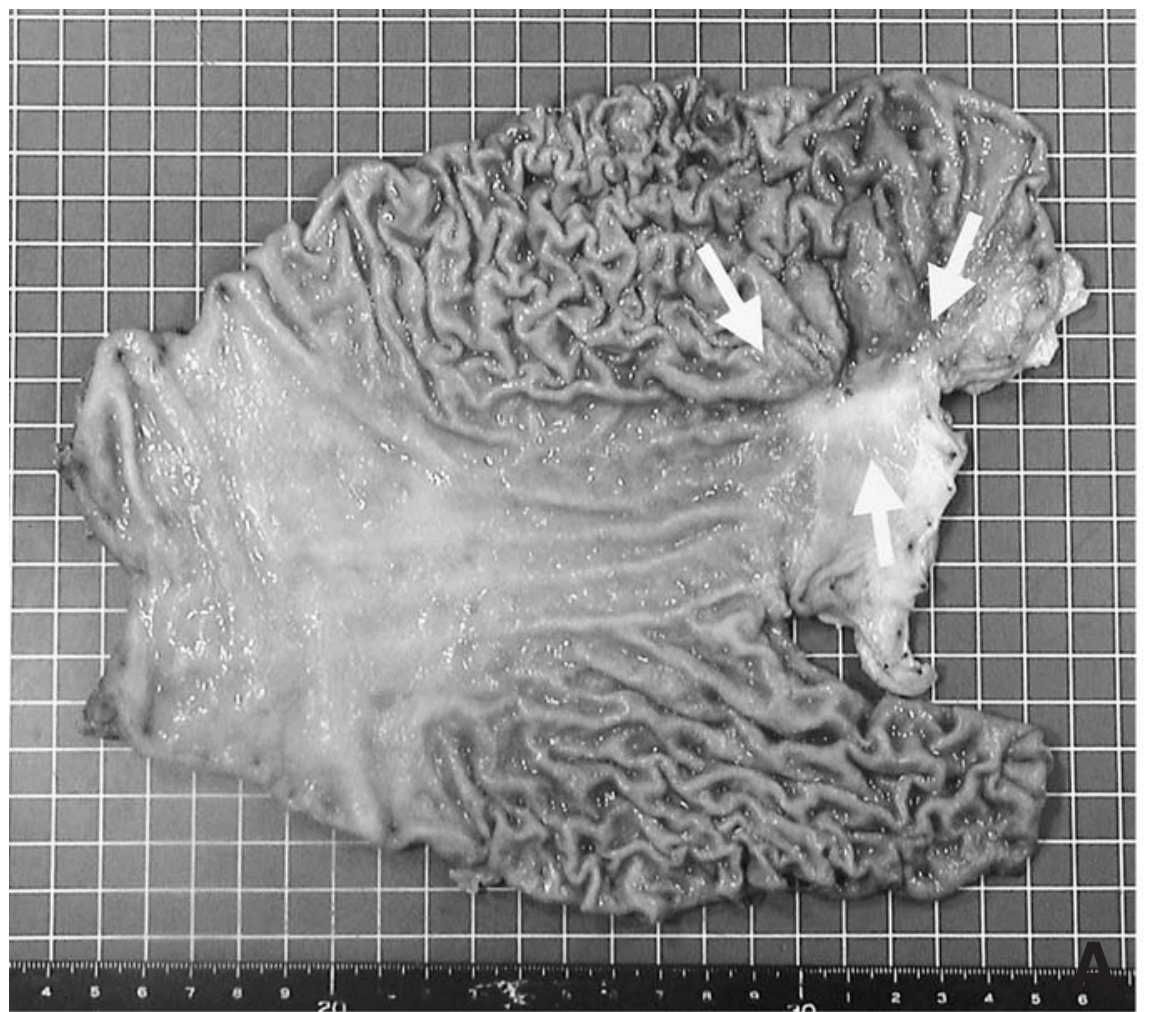

Fig. 4. A The surgically resected stomach. There is neither an ulcerated lesion nor a tumor. The upper part of the stomach showed sclerotic changes with fibrosis (arrows). B The histological appearance indicated an effective response to chemotherapy in the residual tumors. No tumor cells were observed in any lesions of the primary tumor (arrows), leading to a complete response (CR) diagnosis. $\mathbf{C}$ The histology also indicated a positive chemotherapeutic response in the residual lymph nodes. Strong fibrosis with lymphocytic infiltrations and foreign body granuloma were observed, and this region was also diagnosed as showing $\mathrm{CR}$. B H\&E, $\times 100$; C H\&E, $\times 100$
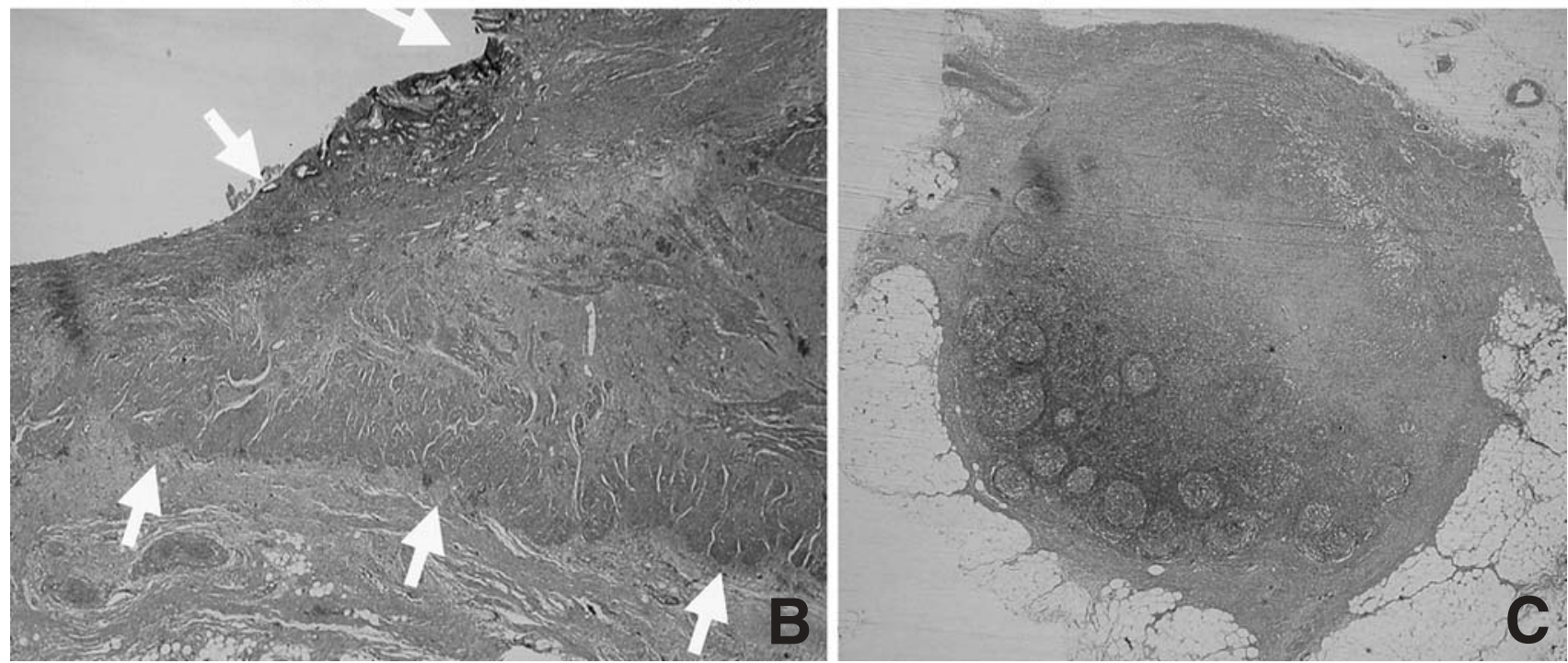

tion, in response to several regimens, have been reported frequently. On the other hand, a CR confirmed by histological examination is rarely found in gastric carcinomas, although Iwahashi et al. [16] reported a case of a histological CR obtained by means of an S-1 and CDDP combination. Although we are not sure whether the prognosis, even for these CR patients, is excellent, we hope to demonstrate that the chemoradiotherapy shows potent efficacy as a first-line treatment for highly advanced gastric carcinoma. While we found grade 3 toxic effects produced by the first regimen, with $20 \mathrm{mg} / \mathrm{m}^{2}$ per day of CDDP, the microscopic CR brought about by the regimen might improve the poor prognosis associated with highly advanced gastric carcinoma.

\section{References}

1. Hundahl SA, Phillips JL, Menck HR. The National Cancer Data Base report on poor survival of U.S. gastric carcinoma patients treated with gastrectomy: fifth edition. American joint committee 
on cancer staging, proximal disease, and the "different disease" hypothesis. Cancer 2000;88:921-32.

2. Landry J, Tepper JE, Wood WC, Moulton EO, Koerner F, Sullinger J. Patterns of failure following curative resection of gastric cancer. Int J Radiat Oncol Biol Phys 1990;191:135762.

3. Ochiai T, Hayashi H, Suzuki $T$, Nakajima $K$, Shimada $H$, Hishikawa E, et al. Evaluation of a new staging system by the Japanese Research Society for Gastric Cancer. Surg Today 1998;28:1015-21.

4. Macdonald JS, Smalley SR, Benedetti J, Hundahl SA, Estes NC, Stermmermann GN, et al. Chemoradiotherapy after surgery compared with surgery alone for adenocarcinoma of the stomach or gastroesophageal junction. N Engl J Med 2001;345:72530.

5. Waters JS, Norman A, Cunningham D, Scarffe JH, Webb A, Herper $\mathrm{P}$, et al. Long-term survival after epirubicin, cisplatin and fluorouracil for gastric cancer: results of a randomized trial. Br J Cancer 1999;80:269-72.

6. Vanhoefer U, Rougier P, Wilke H, Ducreux MP, Lacave AJ, Van Cutsem E, et al. Final results of a randomized phase III trial of sequential high-dose methotrexate, fluorouracil, and doxorubicin versus etoposide, leucovorin, and fluorouracil versus infusional fluorouracil and cisplatin in advanced gastric cancer: a trial of the European Organization for Research and Treatment of Cancer Gastrointestinal Trace Cancer Cooperative Group. J Clin Oncol 2000;18:2648-57.

7. Ravaud A, Borner M, Schellens JH, Geoffrois L, Schoffski BP, Kroon K, et al. EORTC-ECSG. UFT and leucovorin in first-line chemotherapy for patients with metastatic gastric cancer. An Early Clinical Studies Group (ECSG)/European Organization for Research and Treatment of Cancer (EORTC) phase II trial. Eur J Cancer 2001;37:1642-7.

8. Garcia AA, Leichman CG, Lenz HJ, Baranda J, Lujan R, Casagrande Y, Leichman L. Phase II trial of outpatient schedule of paclitaxel in patients with previously untreated metastatic, measurable adenocarcinoma of the stomach. Jpn J Clin Oncol 2001;31:275-8

9. Harris BE, Song R, Soong ST, Diasio RB. Relationship between dihydropyrimidine dehydrogenase activity and plasma drug levels in cancer patients receiving 5-fluorouracil by protracted continuous infusion. Cancer Res 1990;50:197-201.
10. Shirasaka T, Shimamoto Y, Ohshimo H, Yamaguchi M, Kato T, Yonekura K, Fukushima M. Development of a novel form of an oral 5-fluorouracil derivative (S-1) directed to the potentiation of the tumor selective cytotoxicity of 5-fluorouracil by two biochemical modulators. Anticancer Drugs 1996;7:548-57.

11. Shirasaka K, Shimamoto Y, Fukushima M. Inhibition by oxonic acid of gastrointestinal toxicity of 5-fluorouracil without loss of its antitumor activity in rats. Cancer Res 1993;53:4004-9.

12. Sugimachi K, Maehara Y, Horikoshi N, Shimada Y, Sakata Y, Mitachi Y, Taguchi T. An early phase II study of oral S-1, a newly developed 5-fluorouracil derivative for advanced and recurrent gastrointestinal cancers. The S-1 Gastrointestinal Cancer Study Group. Oncology 1999;57:202-10.

13. Sakata Y, Ohtsu A, Horikoshi N, Sugimachi K, Mitachi Y, Taguchi T. Late phase II study of novel oral fluoropyrimidine anticancer drug S-1 (1 M tegafur- $0.4 \mathrm{M}$ gimestat-1 M otastat potassium) in advanced gastric cancer patients. Eur J Cancer 1998;34:1715-20.

14. Chung YS, Yamashita Y, Inoue T, Matsuoka T, Nakata B, Onoda $\mathrm{N}$, et al. Continuous infusion of 5-fluorouracil and low dose cisplatin infusion for the treatment of advanced and recurrent gastric adenocarcinoma. Cancer 1997;80:1-7.

15. Shirasaka T, Yamamitsu S, Tsuji A, Taguchi T. Conceptual changes in cancer chemotherapy: from an oral fluoropyrimidine prodrug, UFT, to a novel oral fluoropyrimidine prodrug, S-1, and low-dose FP therapy in Japan. Invest New Drugs 2000;18:315-29.

16. Iwahashi M, Nakamori M, Tani M, Yamaue H, Sakaguchi S, Nakamura M, et al. Complete response of highly advanced gastric cancer with peritoneal dissemination after new combined chemotherapy of S-1 and low dose cisplatin: report of a case. Oncology 2001;61:16-22.

17. Japanese Research Society for Gastric Cancer. Japanese classification of gastric carcinoma. 16th Ed. Tokyo: Kanehara; 2001.

18. Official reference of CTC version 2.0: Cancer Therapy Evaluation Program. Common Toxicity Criteria, Version 2.0. DCTD, NCI, NIH, DHHS 1998.

19. Miller AB, Hoogstraten B, Staquet M, Winkler A. Reporting results of cancer treatment. Cancer 1981;47:207-14.

20. Saikawa Y, Akasaka Y, Kanai T, Otani Y, Kumai K, Kubota T, Kitajima M. Preoperative combination chemotherapy with S-1 and low dose cisplatin against highly advanced gastric carcinoma. Oncol Rep 2003;10:381-6. 